\title{
New electron energy transfer and cooling rates by excitation of $\mathrm{O}_{2}$
}

\author{
A. V. Pavlov \\ Institute of Terrestrial Magnetism, Ionosphere and Radio-Wave Propagation, Russia Academy of Science (IZMIRAN), Troitsk, \\ Moscow Region, 142092, Russia
}

Received: 24 September 1997 / Revised: 18 February 1998 / Accepted: 24 February 1998

\begin{abstract}
In this work I present the results of a study of the electron cooling rate, the production rates of vibrationally excited $\mathrm{O}_{2}$, and the production frequency of the $\mathrm{O}_{2}$ vibrational quanta arising from the collisions of electrons with $\mathrm{O}_{2}$ molecules as functions of the electron temperature. The electron energy transfer and cooling rates by vibrational excitation of $\mathrm{O}_{2}$ have been calculated and fitted to analytical expressions by use of the revised vibrationally excited $\mathrm{O}_{2}$ cross sections. These new analytical expressions are available to the researcher for quick reference and accurate computer modeling with a minimum of calculations. It is also shown that the currently accepted rate of electron energy loss associated with rotational transitions in $\mathrm{O}_{2}$ must be decreased by a factor of 13 .
\end{abstract}

\section{Introduction}

The electron temperature in the ionosphere is of great significance in that it usually controls the rates of many physical and chemical ionospheric processes. The theoretical computation of electron temperature distribution in the ionosphere requires the knowledge of various heating and cooling rates. Lane and Dalgarno (1969) have shown that the electron cooling by excitation of $\mathrm{O}_{2}$ should be important in the E-region of the ionosphere (we shall use the symbol $\mathrm{O}_{2}$ for the ground electronic state $\mathrm{X}^{3} \sum_{g}^{-}$of $\mathrm{O}_{2}$ ). Hierl et al. (1997) found that the big difference between the high temperature flowing afterglow and drift tube measurements (McFarland et al., 1973; Albritton et al., 1977) of the effective rate coefficient for the reaction of $\mathrm{O}^{+}\left({ }^{4} \mathrm{~S}\right)$ with $\mathrm{O}_{2}$ results from the input of the reactions between the vibrationally excited $\mathrm{O}_{2}$ and $\mathrm{O}^{+}\left({ }^{4} \mathrm{~S}\right)$. Pavlov et al. (1998) and Pavlov (1998) found that at solar maximum and moderate solar-activity conditions the increase in the $\mathrm{O}^{+}\left({ }^{4} \mathrm{~S}\right)+\mathrm{O}_{2}$ loss rate due to vibrationally excited $\mathrm{O}_{2}$ produces up to $40 \%$ reductions in the calculated daytime F2 peak electron density if the vibrational temperature of $\mathrm{O}_{2}$ is the same as the neutral translation temperature. To calculate real values of the $\mathrm{O}_{2}$ vibrational temperatures and the effects of vibrationally excited $\mathrm{O}_{2}$ on the calculated electron density the knowledge of the energy transfer rates from thermal electrons to vibrationally excited $\mathrm{O}_{2}$ is required.

The energy transfer rates from electrons to $\mathrm{O}_{2}$ have been calculated and fitted to analytical expressions by Prasad and Furman (1973) for vibrational and low-lying electronic excitation of $\mathrm{O}_{2}$. These electron cooling rates due to vibrational excitation of $\mathrm{O}_{2}$ and excitation of ${ }^{1} \Delta_{q}$ and ${ }^{1} \sum_{q}^{+}$states of $\mathrm{O}_{2}$ which are currently used in models of the 1onosphere are based on the pioneering $\mathrm{O}_{2}$ vibrational and electronic cross sections of Linder and Schmidt (1971). However, Lawton and Phelps (1978) found that they have to increase the $\mathrm{O}_{2}$ energy integrated vibrational cross sections of Linder and Schmidt (1971) by a factor of 2 to obtain satisfactory agreement with their measured electron transport coefficients. The recent measurements of the energy integrated cross sections of Allan (1995) are larger than those given by Linder and Schmidt (1971) by a factor of nearly 3. Linder and Schmidt (1971) measured the energy integrated cross sections only for excitation of $\mathrm{O}_{2}$ at the vibrational levels $v=1,2,3,4$ while Allan (1995) presented these cross sections for vibrational excitation of the $v=0 \rightarrow$ $1,2,3,4,5,6,7$ levels by electrons with energies in the range from threshold to $3 \mathrm{eV}$ where the ${ }^{2} \Pi_{g}$ resonance dominates. Furthermore, as a result of the improved sensitivity and resolution of the instrument, the number of the narrow resonance peaks given by Allan (1995) for each vibrational level $v=1,2,3,4$ is larger than those obtained by Linder and Schmidt (1971). The primary object of this study is to use the $\mathrm{O}_{2}$ energy integrated vibrational cross sections measured by Allan (1995) to calculate and to fit to new analytical expressions for energy transfer rates from electrons to $\mathrm{O}_{2}$. 
As far as the author knows, rotational excitation cross sections of $\mathrm{O}_{2}$ have not been measured. To calculate the electron cooling rate due to rotational excitation of $\mathrm{O}_{2}$ by electron impact the quadrupole Born approximation formula of Mentzoni and Row (1963) with theoretical rotational excitation cross sections given by Gerjuoy and Stein (1955) is usually used. As a result, the electron cooling rate given by Dalgarno et al. (1968) with an effective quadrupole moment of Hake and Phelps (1967) is currently used in models of the ionosphere. The accuracy of this approach is discussed in our work on the basis of the Smith and Dean (1975) measurements of the electron cooling rate in $\mathrm{O}_{2}$ gas.

\section{Electron energy transfer rates for vibrational excitation of $\mathrm{O}_{2}$}

During the vibrational excitation of the unexcited $\mathrm{O}_{2}(v=0)$ by electron impact the temporary capture of the electron occurs to form the excited ion $\mathrm{O}_{2}^{-2} \Pi_{g}$ at vibrational level, $j$, with the following vibrational excitation of $\mathrm{O}_{2}$ :

$\mathrm{O}_{2}(v=0)+\mathrm{e} \leftrightarrow \mathrm{O}_{2}^{-2} \Pi_{g}(j>4) \leftrightarrow \mathrm{O}_{2}(v>0)+\mathrm{e}$.

The electron cooling rate arising from the collisions of thermal electrons with $\mathrm{O}_{2}(v=0)$ can be calculated as

$L(v=0)=N_{e} \sum_{v=1}^{7} \mathrm{Q}_{0 v}\left\{\left[\mathrm{O}_{2}(0)\right]-\left[\mathrm{O}_{2}(v)\right] \exp \left(E_{v} T_{e}^{-1}\right)\right\}$

where

$\mathrm{Q}_{0 v}=E_{v}\left\{8 k T_{e}\left(\pi m_{e}\right)^{-1}\right\}^{0.5} \int_{0}^{\infty} \sigma_{0 v}(x) x \exp (-x) \mathrm{d} x$,

$E_{v}=v E_{1}-v(v-1) \Delta E, x=E\left(k T_{e}\right)^{-1}, E$ is the energy of electrons, $E_{v}$ is the energy of the $v$-th vibrational level, $\Delta E=17.3 \mathrm{~K}$ is anharmonicity of $\mathrm{O}_{2}$ (Radzig and Smirnov, 1980), $E_{1}=2239 \mathrm{~K}$ (or $0.1929 \mathrm{eV}$ ) is the energy of the first vibrational level of $\mathrm{O}_{2}$ given by Radzig and Smirnov (1980), $\sigma_{0 v}(E)$ are the partial cross sections for excitation of $\mathrm{O}_{2}(0)$ by electrons, $k$ is Boltzmann's coefficient, and $m_{e}$ denotes the mass of electrons.

Allan (1995) found very sharp peaks in the vibrational cross sections in the resonance region $0.3-3.0 \mathrm{eV}$ of $\mathrm{O}_{2}$ and could determine the cross sections integrated over a certain range of energy for vibrational excitation of $\mathrm{O}_{2}$. The use of the integral vibrational-excitation cross sections of Allan (1995) in these narrow spikes leads to

$Q_{0 v}=E_{v} \sqrt{8}\left(k T_{e} \pi m_{e}\right)^{-0.5} \sum_{j=5}^{24} S_{j v} X_{j} \exp \left(-X_{j}\right)$

where $j$ is the number of the $\mathrm{O}_{2}^{-} 2 \Pi_{g}$ vibrational level, $S_{j v}$ is the integral vibrational-excitation cross section for the sharp peak in the vibrational cross section at the energy $E_{j}^{*}, X_{j}=E_{j}^{*}\left(k T_{e}\right)^{-1}, E_{j}^{*}$ is the energy of the incident electron at the centre of the $\mathrm{O}_{2}^{-} 2 \Pi_{g}$ resonance given by Allan (1995), the energy resolution is not sufficient to resolve rotational structure and the discussed measurements of cross sections yield rotationally averaged vibrational-excitation cross sections.

Equation (2) includes both excitation and deexcitation, which are related through the principle of detailed balancing. To calculate $\left[\mathrm{O}_{2}(v)\right]$ it is necessary to solve the vibrational quanta continuity equation [a Boltzmann distribution of $\mathrm{O}_{2}(v)$ ] or the full continuity equations for densities of $\mathrm{O}_{2}(v)$ [a non-Boltzmann distribution of $\left.\mathrm{O}_{2}(v)\right]$. The system of full continuity equations and the vibrational quanta continuity equation are described by Pavlov $(1989,1994,1998)$ using the harmonic and anharmonic oscillator energy level approximations, the vibrational-vibrational and vibrational-translational energy exchange of $\mathrm{O}_{2}(v)$, the diffusion of $\mathrm{O}_{2}(v)$ in the mixture of $\mathrm{O}_{2}(0), \mathrm{N}_{2}$, and $\mathrm{O}$, and the production rates of $\mathrm{O}_{2}(v)$. The values of the production rates of $\mathrm{O}_{2}(v)$ due to the thermal electron excitation of $\mathrm{O}_{2}(0)$ and the deexcitation of $\mathrm{O}_{2}(v)$ in the reaction (1) can be calculated as

$q_{v}=N_{e} Q_{0 v} E_{v}^{-1}\left\{\left[\mathrm{O}_{2}(0)\right]-\left[\mathrm{O}_{2}(v)\right] \exp \left(E_{v} T_{e}^{-1}\right)\right\}$

where $Q_{0 v}$ is the same as in Eq. (4).

Pavlov (1998) found that during the undisturbed and geomagnetic storm periods at middle latitudes at moderate solar-activity conditions deviation from a Boltzmann distribution of $\mathrm{O}_{2}(v)$ was large at the vibrational energy levels $v>3$ and close to a Boltzmann distribution of $\mathrm{O}_{2}(v)$ for $v=1,2$, and 3. The main contribution to $L(v=0)$ arising from the deexcitation of $\mathrm{O}_{2}(v)$ is brought by the deexcitation of $\mathrm{O}_{2}(1)$ and $\mathrm{O}_{2}(2)$. As a result, it is possible to use a Boltzmann distribution of $\mathrm{O}_{2}(v)$ in calculations of $L(v=0)$ as

$$
\begin{aligned}
{\left[\mathrm{O}_{2}(v)\right] } & =\left[\mathrm{O}_{2}(0)\right]\left\{\alpha(1+\alpha)^{-1}\right\}^{v} \\
& =\left[\mathrm{O}_{2}(0)\right] \exp \left(-v E_{1} / T_{\mathrm{vib}}\right),
\end{aligned}
$$

where the vibrational quanta is defined as $\alpha=\sum_{v=1}^{\infty} v\left[\mathrm{O}_{2}(v)\right] /\left[\mathrm{O}_{2}\right]$, and the vibrational temperature is defined as $T_{\mathrm{vib}}=-E_{1} / \ln \left[\alpha(1+\alpha)^{-1}\right]$.

In this case from Eq. (6) it follows that the sum of the vibrationally unexcited and excited molecular oxygen concentrations is

$\left[\mathrm{O}_{2}\right]=\sum_{v=0}^{\infty}\left[\mathrm{O}_{2}(v)\right]=\left[\mathrm{O}_{2}(0)\right](1+\alpha)$.

There is no possibility to differentiate the vibrationally unexcited $\mathrm{O}_{2}$ from the vibrationally excited $\mathrm{O}_{2}$ during the mass-spectrometer measurements of the neutral species in the atmosphere, and it is necessary to point out that the MSIS-86 model (Hedin, 1987) which is usually used in most models calculates the value of $\left[\mathrm{O}_{2}\right]$. The difference between $\left[\mathrm{O}_{2}\right]$ and $\left[\mathrm{O}_{2}(0)\right]$ is not negligible during solar maximum. For example, $\left[\mathrm{O}_{2}\right] /\left[\mathrm{O}_{2}(0)\right]=$ 1.29 for $T_{\mathrm{vib}}=1500 \mathrm{~K}$.

The total electron cooling rate associated with vibrational transitions of $\mathrm{O}_{2}$ arises from the collisions of thermal electrons with unexcited $\mathrm{O}_{2}(v=0)$ and vibrationally excited $\mathrm{O}_{2}(v>0)$ molecules. The value of this rate is defined as $L_{\mathrm{vib}}\left(\mathrm{O}_{2}\right)=\sum_{v=0}^{\infty} L(v)$. As far as the 
author knows, vibrational excitation cross sections of $\mathrm{O}_{2}(v>0)$ by electron impact have not been measured and not calculated from theory. To simplify this problem Prasad and Furman (1973) did not distinguish between vibrational excitation cross sections of $\mathrm{O}_{2}(v=0)$ and $\mathrm{O}_{2}(v>0)$. In this approximation for the Boltzmann distribution given by Eq. (6) the total rate of electron energy loss associated with vibrational transitions of $\mathrm{O}_{2}(v)$ is

$L_{\mathrm{vib}}\left(\mathrm{O}_{2}\right)=N_{e}\left[\mathrm{O}_{2}\right] \sum_{v=1}^{7} Q_{0 v}\left\{1-\exp \left[v E_{1}\left(T_{e}^{-1}-T_{\mathrm{vib}}^{-1}\right)\right]\right\}$.

The number density of vibrationally unexcited $\mathrm{O}_{2}$ is much larger than the number density of vibrationally excited $\mathrm{O}_{2}$ during the undisturbed and geomagnetic storm periods at middle latitudes (Pavlov, 1989, 1994, 1998). As a result, the collisions of thermal electrons with vibrationally unexcited $\mathrm{O}_{2}$ yield the main contribution to $L_{\mathrm{vib}}\left(\mathrm{O}_{2}\right)$ and the possible error of Eq. (8) is expected to be small.

To calculate $\alpha$ or $T_{\mathrm{vib}}$ it is necessary to solve the vibrational quanta continuity equation (Pavlov, 1989, 1994, 1998). The vibrational-translational energy exchange of $\mathrm{O}_{2}(v)$, the diffusion of $\mathrm{O}_{2}(v)$ in the mixture of $\mathrm{O}_{2}(0), \mathrm{N}_{2}$, and $\mathrm{O}$, and the production frequencies of $\alpha$ are taken into account in the vibrational quanta continuity equation. The values of the production frequency, $W$, of the vibrational quanta due to the thermal electron excitation of $\mathrm{O}_{2}(0)$ and the deexcitation of $\mathrm{N}_{2}(v)$ can be calculated as $W=L(v=0)\left\{E_{1}\left[\mathrm{O}_{2}\right]\right\}^{-1}$.

Prasad and Furman (1973) found the analytical expressions for $Q_{0 v}(P F)$ based on the $\mathrm{O}_{2}$ integral vibrational cross sections of Linder and Schmidt (1971) for the vibrational levels $v=1,2$ and 3 and we can compare their results with $Q_{0 v}$ based on the Allan (1995) cross sections.
Solid lines on Fig. 1 show $Q_{0 v}$ as functions of $T_{e}$ and $v$. Curves $1,2, \ldots 7$ on Fig. 1 are the calculated $Q_{0 v}$ for $v=1,2, \ldots 7$ of $\mathrm{O}_{2}(v)$. Dashed lines 1, 2 and 3 on Fig. 1 show $Q_{0 v}(P F)$ for $v=1,2$ and 3 of $\mathrm{O}_{2}(v)$. It is evident that the energy transfer rates obtained by using the Allan (1995) cross sections are much larger than those obtained by using the Linder and Schmidt (1971) data within the electron temperature range 300-6000 K: $Q_{01} / Q_{01}(P F)=4.9-12.4, Q_{02} / Q_{02}(P F)=4.0-10.6$, and $Q_{03} / Q_{04}(P F)=4.6-69.1$. These results show the importance of the use of the integral vibrational cross sections of Allan (1995) instead of those from Linder and Schmidt (1971) in calculations of the production rates of $\mathrm{O}_{2}(v)$ due to the thermal electron excitation of $\mathrm{O}_{2}(0)$.

The total cooling rate by vibrational excitation of $\mathrm{O}_{2}$ is given by Prasad and Furman (1973) as

$L(P F)=N_{e}\left[\mathrm{O}_{2}\right] U\left(T_{e}\right)\left\{1-\exp \left[2770\left(T_{e}^{-1}-T_{n}^{-1}\right)\right]\right\}$,

where $U\left(T_{e}\right)=5.196 \cdot 10^{-13} \exp \left[f_{4}\left(1 / 700-1 / T_{e}\right)\right], f_{4}=$ $3300-839 \sin \left[0.000191\left(T_{e}-2700\right)\right], T_{n}$ is a neutral temperature, the units of $T_{e}$ and $T_{n}$ are $\mathrm{K}$ and the units of $\mathrm{U}\left(T_{e}\right)$ are $\mathrm{eV} \mathrm{cm} \mathrm{s}^{-1}$. The value of $L(P F)$ includes both excitation $\left(U\left(T_{e}\right)\right)$ and deexcitation $\left(U\left(T_{e}\right) \exp [2770\right.$ $\left.\left.\left(T_{e}^{-1}-T_{n}^{-1}\right)\right]\right)$ of $\mathrm{O}_{2}(v)$. It can been seen from the comparison of Eqs. (8) and (9) that the analytical expression for the cooling rate was found by Prasad and Furman (1973) by assuming that the value of the deexcitation rate of $\mathrm{O}_{2}(v>1)$ is the same as that for $\mathrm{O}_{2}(v=1)$ and $T_{\text {vib }}=T_{n}$. This assumption leads to the most simple approach in calculations of the cooling rate given by Eq. (8) as

$$
L_{\mathrm{vib}}\left(\mathrm{O}_{2}\right)=N_{e}\left[\mathrm{O}_{2}\right] Q\left(T_{e}\right)\left\{1-\exp \left[E_{1}\left(T_{e}^{-1}-T_{n}^{-1}\right)\right]\right\},
$$

where $Q\left(T_{e}\right)=\sum_{v=1}^{7} Q_{0 v}\left(T_{e}\right)$.

To compare $L_{\mathrm{vib}}\left(\mathrm{O}_{2}\right)$ with $L(P F)$ it is necessary to compare $U\left(T_{e}\right)$ with $Q\left(T_{e}\right)$. The calculated values of

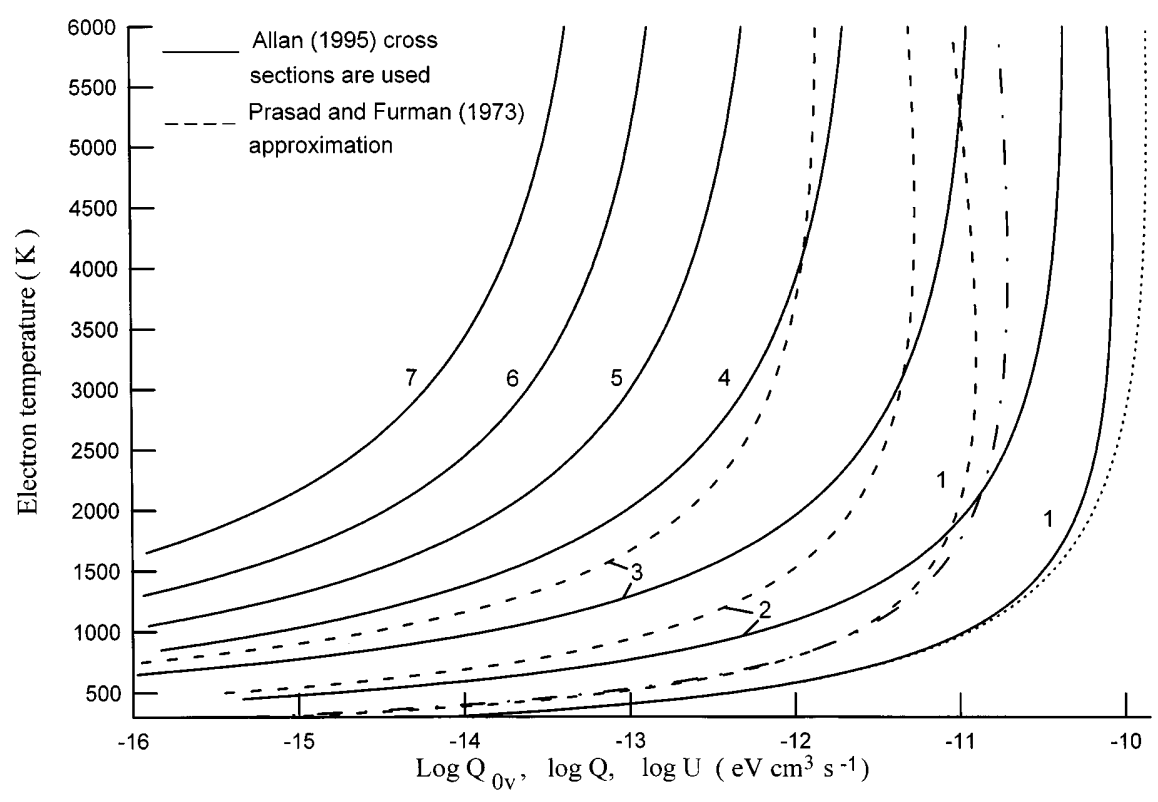

Fig. 1. Comparison of $\mathrm{Q}_{0 \mathrm{v}}$ calculated by use of the energy integrated cross sections of Allan (1995) (solid lines) and Linder and Schmidt (1971) (dashed lines) for the $\mathrm{O}_{2}$ vibrational levels $v=1,2, \ldots 7$ (curves $1,2, \ldots 7)$. Dotted line is $Q\left(T_{e}\right)=\sum_{v=1}^{7} Q_{0 v}\left(T_{e}\right)$ calculated in this study by using the energy integrated cross sections of Allan (1995). Dashed-dotted line is $U\left(T_{e}\right)=\sum_{v=1}^{4} Q_{0 v}\left(T_{e}\right)$ given by Prasad and Furman (1973) and based on the $\mathrm{O}_{2}$ integral vibrational cross sections of Linder and Schmidt (1971) 
$U\left(T_{e}\right)$ and $Q\left(T_{e}\right)$ are shown in Fig. 1 as dashed-dotted and dotted lines. The value of the ratio $U\left(T_{e}\right) / Q\left(T_{e}\right)$ is about 4.7-17.9 within the electron temperature range $300-6000 \mathrm{~K}$. We can see that the use of the Prasad and Furman (1973) approach leads to very serious error in the cooling rate by vibrational excitation of $\mathrm{O}_{2}$.

The value of $Q_{0 v}$ increases with increasing the number of vibrational level. The relative contribution to $L$ and $W$ of the upper vibrational levels can be evaluated from the ratio $R_{k}=\sum_{v=k}^{7} Q_{0 v}\left(T_{e}\right) / Q\left(T_{e}\right)$. The value of $R_{5}$ is less than 0.005 and the value of $R_{4}$ is less than 0.02 within the electron temperature range 300-6000 K. Therefore the vibrational levels $v=1-4$ are enough to

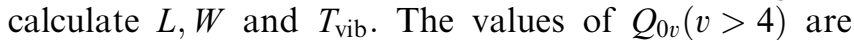
necessary to calculate $\left[\mathrm{O}_{2}(v>4)\right]$ by solving the full continuity equations for densities of $\mathrm{O}_{2}(v)$ given by Pavlov (1998) (a non-Boltzmann distribution of $\mathrm{O}_{2}(v)$ ).

Wong et al. (1973), Shyn and Sweeney (1993), Allan (1995) and Noble et al. (1996) found the broad maximum in the vibrational cross sections of $\mathrm{O}_{2}$ near $10 \mathrm{eV}$ in addition to the sharp peaks below about $2.5 \mathrm{eV}$ arising from the ${ }^{2} \Pi_{g}$ resonance of the reaction (1). The ${ }^{2} \Pi_{g}$ resonance dominates the vibrational cross sections in the ground electronic state of $\mathrm{O}_{2}$ at low energies below about $2.5 \mathrm{eV}$ while the ${ }^{4} \sum_{u}^{-}$and ${ }^{2} \sum_{u}^{-}$resonances of $\mathrm{O}_{2}$ give the major contribution to the $\mathrm{O}_{2}$ vibrational cross sections above 3-4 eV (Allan, 1995; Noble et al., 1996).

Let us denote the value of $Q_{0 v}$ for the ${ }^{2} \Pi_{g}$ resonance as $Q_{0 v}(\Pi)\left(Q_{0 v}(\Pi)\right.$ is presented in Fig. 1 as a function of $T_{e}$ for the each vibrational level $v=1-7$ ) and the value of $Q_{0 v}$ for the ${ }^{4} \sum_{u}^{-}$and ${ }^{2} \sum_{u}^{-}$resonances as $Q_{0 v}\left(\sum\right)$. To calculate $Q_{0 v}\left(\sum\right)$ from Eq. (3) we used the measured $\sigma_{0 v}(E)$ of Noble et al. (1996) for the electron energy range $5-15 \mathrm{eV}$. We also used the differential cross sections, $\Omega_{0 v}$, given by Allan (1995) and $\sigma_{0 v}\left(E^{*}=5-7 \mathrm{eV}\right)$ measured by Noble et al. (1996) to calculate $\sigma_{0 v}(E)=\sigma_{0 v}\left(E^{*}\right) \Omega_{0 v}(E) / \Omega_{0 v}\left(E^{*}\right)$ for the region of electron energy from $3 \mathrm{eV}$ to $5 \mathrm{eV}$. As a result, the ratio $Q_{0 v}\left(\sum\right) / Q_{0 v}(\Pi)$ is less than 0.005 for $v=1-4$ within the electron temperature range $300-5000 \mathrm{~K}$ and the effects of the ${ }^{4} \sum_{u}^{-}$and ${ }^{2} \sum_{u}^{-}$resonances on $Q_{0 v}$ are negligible in comparison with those for the ${ }^{2} \Pi_{g}$ resonance.

The electron cooling rate, the production rates of $\mathrm{O}_{2}(v)$, and the production frequency of the $\mathrm{O}_{2}$ vibrational quanta depend on $Q_{0 v}$ which is a function of the electron temperature. We now give an analytical expression, $Q_{0 v}^{*}$, for $Q_{0 v}$ available to the researcher for quick reference and accurate computer modeling with a minimum of calculations as

$$
Q_{0 v}^{*}=A_{v} \exp \left\{\left(1-B_{v} T_{e}^{-1}\right)\left(C_{v}+D_{v} \sin \left[F_{v}\left(T_{e}-G_{v}\right)\right]\right)\right\},
$$

where the constants $A_{v}, B_{v}, C_{v}, D_{v}, F_{v}$, and $G_{v}$ are given in Table 1.

To establish how good or bad our analytical expression can be we define quantitative measure of maximum error of analytical approach of Eq. (11) for given range of $T_{e}$ as $\delta_{v}=\max \left|1-Q_{0 v}^{*} / Q_{0 v}\right|$. The calculated values of $\delta_{v}$ are presented in Table 1 for the electron temperature range from $300-340 \mathrm{~K}$ to $3950-5650 \mathrm{~K}$ where Eq. (11) is recommended for use. The above analytical expressions reproduce the numerically calculated $Q_{0 v}$ within $9-14 \%$. The measured energy integrated cross sections are believed to be accurate within $\pm 35 \%$., except small amount of data where the error can be up to a factor of 2 (Allan, 1995). Therefore the accuracy within 9-14\% given in Table 1 is enough to reproduce $Q_{0 v}$.

To use Eq. (10) in calculations of the cooling rate by vibrational excitation of $\mathrm{O}_{2}$ we obtained an analytical expression, $Q^{*}\left(T_{e}\right)$, for $Q\left(T_{e}\right)$ as

$$
\begin{aligned}
Q^{*}\left(T_{e}\right)= & 8.8 \cdot 10^{-15} \exp \left\{\left(1-304 T_{e}^{-1}\right)\right. \\
& \left.\times\left(10.2+0.4 \sin \left[1.083 \cdot 10^{-3}\left(T_{e}-1000\right)\right]\right)\right\}, \\
& \quad \mathrm{eVcm}^{3} \mathrm{~s}^{-1} .
\end{aligned}
$$

The value of maximum error for the electron cooling rate may be evaluated as $\delta=\max \left|1-Q^{*}\left(T_{e}\right) / Q\left(T_{e}\right)\right|$. We found that $\delta=0.10$ within the electron temperature range $300-3600 \mathrm{~K}$ and this accuracy is enough to reproduce $L_{\mathrm{vib}}\left(\mathrm{O}_{2}\right)$.

\section{The electron cooling rate by low-lying electronic excitation of $\mathrm{O}_{2}$}

There are several independent measurements of the integral excitation cross sections for the lower lying excited states of $\mathrm{O}_{2}\left(a^{1} \Delta_{g}\right)$ and $\mathrm{O}_{2}\left(b^{1} \sum_{g}^{+}\right)$in the electron energy range up to 4-20 eV. Linder and Schmidt (1971) reported the integral cross sections for electronic excitation of the $a^{1} \Delta_{g}$ and $b^{1} \sum_{g}^{+}$states with electrons of incident energies in the range from thresholds to $4 \mathrm{eV}$ while Trajmar et al. (1971) measured these cross sections in the energy range $4-45 \mathrm{eV}$. The most recent measurements of the $a^{1} \Delta_{g}$ and $b^{1} \sum_{g}^{+}$integral cross sections were

Table 1. Coefficients for calculations of $Q_{0 v}$

\begin{tabular}{llllllll}
\hline $\mathrm{v}$ & $A_{v}, \mathrm{eVcm}^{3} \mathrm{~s}^{-1}$ & $B_{v}, K$ & $C_{v}$ & $D_{v}$ & $F_{v}$ & $\mathrm{G}_{\mathrm{v}}, \mathrm{K}$ & $\delta_{\mathrm{v}}$ \\
\hline 1. & $8.56 \cdot 10^{-15}$ & 303 & 10.00 & -0.20 & $1.05 \cdot 10^{-3}$ & 3150 & $0.11\left(300 \mathrm{~K} \leq T_{e} \leq 5400 \mathrm{~K}\right)$ \\
2. & $1.15 \cdot 10^{-18}$ & 305 & 18.78 & -0.25 & $9.24 \cdot 10^{-4}$ & 3450 & $0.11\left(340 \mathrm{~K} \leq T_{e} \leq 5650 \mathrm{~K}\right)$ \\
3. & $1.77 \cdot 10^{-23}$ & 301 & 29.00 & -0.20 & $6.16 \cdot 10^{-4}$ & 3150 & $0.11\left(300 \mathrm{~K} \leq T_{e} \leq 5650 \mathrm{~K}\right)$ \\
4. & $7.05 \cdot 10^{-25}$ & 301 & 30.07 & 0.31 & $1.00 \cdot 10^{-3}$ & 1800 & $0.11\left(300 \mathrm{~K} \leq T_{e} \leq 4550 \mathrm{~K}\right)$ \\
5. & $2.14 \cdot 10^{-28}$ & 299 & 37.52 & -0.42 & $5.28 \cdot 10^{-4}$ & 3000 & $0.11\left(300 \mathrm{~K} \leq T_{e} \leq 4000 \mathrm{~K}\right)$ \\
6. & $2.94 \cdot 10^{-31}$ & 300 & 43.27 & -0.39 & $7.85 \cdot 10^{-4}$ & 3000 & $0.09\left(300 \mathrm{~K} \leq T_{e} \leq 5600 \mathrm{~K}\right)$ \\
7. & $1.08 \cdot 10^{-35}$ & 299 & 53.10 & -0.90 & $5.70 \cdot 10^{-4}$ & 2900 & $0.14\left(315 \mathrm{~K} \leq T_{e} \leq 3950 \mathrm{~K}\right)$ \\
\hline
\end{tabular}


made by Middleton et al. (1992) in the electron energy range 4-45 eV, and Shyn and Sweeney (1993) for electron impact energies 5, 7, 10, and $15 \mathrm{eV}$. These measurements are in good agreement with each other at low energies below about 5-6 eV. The earlier measurements of Trajmar et al. (1971) are in disagreement with the data of Middleton et al.(1992) and Shyn and Sweeney (1993) close to and within the resonance peak region in the cross section of the $a^{1} \Delta_{g}$ and $b^{1} \sum_{g}^{+}$states.

The cooling rates due to the excitation of the $a^{1} \Delta_{q}$ and $b^{1} \sum_{g}^{+}$electronic states of $\mathrm{O}_{2}$ by electron impact have been calculated and fitted to analytical expressions by Prasad and Furman (1973) by using the integral cross sections of Linder and Schmidt (1971). We found that the effects of the excitation of the $a^{1} \Delta_{g}$ and $b^{1} \sum_{g}^{+}$ electronic states of $\mathrm{O}_{2}$ with electrons of incident energies in the range from thresholds to $4 \mathrm{eV}$ give the main contribution to the electron cooling rates. As a result, we can conclude that the generally accepted electron cooling rates of Prasad and Furman (1973) due to the excitation of $\mathrm{O}_{2}\left(a^{1} \Delta_{g}\right)$ and $\mathrm{O}_{2}\left(b^{1} \sum_{g}^{+}\right)$can be used in models of the ionosphere.

The electron cooling rates of Prasad and Furman (1973) includes both excitation and deexcitation of $\mathrm{O}_{2}\left(a^{1} \Delta_{g}\right)$ and $\mathrm{O}_{2}\left(b^{1} \sum_{q}^{+}\right)$, which are related through the principle of detailed balancing. To take into account deexcitation of $\mathrm{O}_{2}\left(a^{1} \Delta_{g}\right)$ and $\mathrm{O}_{2}\left(b^{1} \sum_{g}^{+}\right)$Prasad and Furman (1973) assumed that $\left[\mathrm{O}_{2}\left(a^{1} \Delta_{g}\right)\right]=\left[\mathrm{O}_{2}\left(b^{1} \sum_{g}^{+}\right)\right]$ $=\left[\mathrm{O}_{2}\right] \exp \left(-11400 T_{n}^{-1}\right)$ and $g_{1} / g_{0}=g_{2} / g_{0}=1$ where $g_{0}, g_{1}$, and $g_{2}$ are statistical weights of the electron levels $X^{3} \sum_{g}^{-}, a^{1} \Delta_{g}$, and $b^{1} \sum_{g}^{+}$of $\mathrm{O}_{2}$. After correction of these deexcitation terms the electron cooling rates of Prasad and Furman (1973) are given as

$$
L(\Delta)=N_{e} G\left(T_{e}\right)\left\{\left[\mathrm{O}_{2}\right]-\left[\mathrm{O}_{2}\left(a^{1} \Delta_{g}\right)\right] g_{1} g_{0}^{-1} \exp \left(E_{1} T_{e}^{-1}\right)\right\},
$$

$$
L\left(\sum\right)=N_{e} H\left(T_{e}\right)\left\{\left[\mathrm{O}_{2}\right]-\left[\mathrm{O}_{2}\left(b^{1} \sum_{g}^{+}\right)\right] g_{2} g_{0}^{-1} \exp \left(E_{2} T_{e}^{-1}\right)\right\},
$$

where $\quad G\left(T_{e}\right)=1.143 \cdot 10^{-14} \exp \left[f_{1}\left(1 / 1500-1 / T_{e}\right)\right]$, $H\left(T_{e}\right)=1.616 \cdot 10^{-16} \exp \left[f_{2}\left(1 / 1500-1 / T_{e}\right)\right], E_{1}=11338$ $\mathrm{K}\left(\right.$ or $0.977 \mathrm{eV}$ ) and $E_{2}=18881 \mathrm{~K}$ (or $1.627 \mathrm{eV}$ ) are the energies of the $a^{1} \Delta_{g}$ and $b^{1} \sum_{q}^{+}$levels of $\mathrm{O}_{2}$ (Itikawa et al., 1989), $f_{1}=\left\{13200+1410 \sin \left[0.000241 \quad\left(T_{e}-\right.\right.\right.$ $500)]\} \quad\left\{1+\exp \left[\left(T_{e}-14011\right) / 1048\right]\right\}, \quad f_{2}=\{19225+$ $\left.560 \sin \left[0.000383\left(T_{e}-1000\right)\right]\right\} \quad\left\{1+\exp \left[\left(T_{e}-16382\right) /\right.\right.$ 1760] $\}$, the units of $T_{e}$ are $\mathrm{K}$ and the units of $G\left(T_{e}\right)$ and $H\left(T_{e}\right)$ are $\mathrm{eVcm}^{3} \mathrm{~s}^{-1}, g_{1} / g_{0}=5 / 3$ and $g_{2} / g_{0}=1 / 3$ (Radzig and Smirnov, 1980).

Taking into account the results of observations and calculations of $\mathrm{O}_{2}\left(a^{1} \Delta_{g}\right)$ and $\mathrm{O}_{2}\left(b^{1} \sum_{g}^{+}\right)$emissions and the production and loss rates of $\mathrm{O}_{2}\left(a^{1} \Delta_{g}\right)$ and $\mathrm{O}_{2}\left(b^{1} \sum_{g}^{+}\right)$in chemical reactions given by Lopez-Gonzalez et al. (1989) and Yee et al. (1997) and using the MSIS-86 neutral model (Hedin, 1987) we found that $\left[\mathrm{O}_{2}\right]$ is larger than $\left[\mathrm{O}_{2}\left(a^{1} \Delta_{g}\right)\right] g_{1} g_{0}^{-1} \exp \left(E_{1} T_{e}^{-1}\right)$ or $\left[\mathrm{O}_{2}\left(b^{1} \sum_{g}^{+}\right)\right] g_{2} g_{0}^{-1} \exp \left(E_{2} T_{e}^{-1}\right)$ at the $\mathrm{E}$ and $\mathrm{F}$ region altitudes of the ionosphere. As a result, we can compare
$Q\left(T_{e}\right)$ with $G\left(T_{e}\right)$ and $H\left(T_{e}\right)$ to evaluate the effects of $L(\Delta)$ and $L\left(\sum\right)$ on $T_{e}$ in comparison with the effects of $L_{\text {vib }}\left(\mathrm{O}_{2}\right)$ on $T_{e}$. The resulting values of the ratios $G\left(T_{e}\right) / Q\left(T_{e}\right)$ and $H\left(T_{e}\right) / Q\left(T_{e}\right)$ are less than $3.2 \cdot 10^{-4}$ and $4.5 \cdot 10^{-6}$ for $T_{e} \leq 1500 \mathrm{~K}$. In the electron temperature range 1500-3000 K, we have found that these ratios are less than 0.012 and 0.001 . Thus, we can conclude that the value of $L_{\mathrm{vib}}\left(\mathrm{O}_{2}\right)$ is much larger than the value of $L\left(\sum\right)$ or $L\left(\sum\right)$ and the effects of $L\left(\sum\right)$ and $L\left(\sum\right)$ on the calculation of $T_{e}$ are negligible.

\section{The electron cooling rate by rotational excitation of $\mathrm{O}_{2}$}

The general expression for the rate of electron energy loss associated with rotational transitions in $\mathrm{O}_{2}(v=0)$ is given by Eq. (1) of Stubbe and Varnum (1972). Using the quadrupole Born approximation for rotational excitation cross sections given by Gerjuoy and Stein (1955) and making use of the fact that the rotational states are so close together that the summation can be changed into an integration (the quadrupole Born approximation formula of Mentzoni and Row (1963)), the electron cooling rate by means of rotational excitation of $\mathrm{O}_{2}(v=0)$ can be calculated as

$L_{\mathrm{rot}}\left(\mathrm{O}_{2}(v=0)\right)=\mathrm{C}\left[\mathrm{O}_{2}(v=0)\right] N_{e}\left(T_{e}-T_{n}\right) T_{e}^{-0.5}$,

where $C=\left(64 \pi B a_{0}^{2} Q^{2} 2^{0.5}\right) /\left(15 \pi^{0.5} m_{e}^{0.5}\right), B$ is the rotational constant for the ground vibrational state of $\mathrm{O}_{2}, Q$ is the quadrupole moment averaged over the ground vibrational state of $\mathrm{O}_{2}$, and $a_{0}$ is the Bohr radius.

Dalgarno et al. (1968) used the value of an effective quadrupole moment given by Hake and Phelps (1967) as $Q=-1.8 e a_{0}^{2}$ where $e$ is the electronic charge. This value of $Q$ was chosen by Hake and Phelps (1967) to obtain agreement between the calculated and experimental drift velocities of electrons. As a result, Dalgarno et al. (1968) found $C=7 \cdot 10^{-14} \mathrm{eV} \mathrm{cm} \mathrm{cm}^{-1} \mathrm{~K}^{-0.5}$ (the rotational constant $B=1.793 \cdot 10^{-4} \mathrm{eV}$ given by Radzig and Smirnov (1980) and $Q=-1.8 e a_{0}^{2}$ give more accurate value of $\left.C=6.8 \cdot 10^{-14} \mathrm{eV} \mathrm{cm}^{3} \mathrm{~s}^{-1} \mathrm{~K}^{-0.5}\right)$. However, Lawton and Phelps (1978) assumed later that $Q=-0.3 e a_{0}^{2}$ in their analysis of calculated and measured electron transport coefficients. The value of $C$ is proportional to $Q^{2}$ so that the rate of electron energy loss associated with rotational transitions in $\mathrm{O}_{2}$ given by Dalgarno et al. (1968) should be decreased by a factor of 36.

The cooling rate of electrons in $\mathrm{O}_{2}$ was measured by Smith and Dean (1975) over the electron temperature range $350-800 \mathrm{~K}$. They found that their results are well explained if the quadrupole Born approximation for rotational excitation cross sections with a value of $Q=-0.5 e a_{0}^{2}$ is used. This result is the most practical for us since just the cooling rate of electrons in $\mathrm{O}_{2}$ is studied in this work. As a result of the choice of $Q$ given by Smith and Dean (1975), we can find that $C=5.2 \cdot 10^{-15}$ $\mathrm{eV} \mathrm{cm}{ }^{3} \mathrm{~s}^{-1} \mathrm{~K}^{-0.5}$.

The total rate of electron energy loss associated with rotational transitions within each vibrational level of 
$\mathrm{O}_{2} L_{\text {rot }}\left(\mathrm{O}_{2}\right)=\sum_{v=0}^{\infty} L_{\mathrm{rot}}\left(\mathrm{O}_{2}(v)\right)$. To simplify the problem Dalgarno et al.(1968) used

$L_{\mathrm{rot}}\left(\mathrm{O}_{2}\right)=C\left[\mathrm{O}_{2}\right] N_{e}\left(T_{e}-T_{n}\right) T_{e}^{-0.5}$.

This means that they did not distinguish between rotational excitation cross sections of $\mathrm{O}_{2}(v=0)$ and $\mathrm{O}_{2}(v>0)$. The number density of vibrationally unexcited $\mathrm{O}_{2}$ is much larger than the number density of vibrationally excited $\mathrm{O}_{2}$ during the undisturbed and geomagnetic storm periods at middle latitudes (Pavlov, 1989, 1994, 1998). As a result, the collisions of thermal electrons with vibrationally unexcited $\mathrm{O}_{2}$ yield the main contribution for the rate of electron energy loss associated with rotational transitions within $\mathrm{O}_{2}(v=0)$ and $\mathrm{O}_{2}(v>0)$ and the possible error of Eq. (16) is expected to be small.

Finally, the electron cooling rate due to rotational excitation of $\mathrm{O}_{2}$ by electron impact found in this study is less than that given by Dalgarno et al.(1968) by a factor of 13 .

\section{Conclusions}

The electron energy transfer rates for vibrational excitation of $\mathrm{O}_{2}$ have been calculated and fitted to analytical expressions by the use of the revised vibrationally excited $\mathrm{O}_{2}$ cross sections (Allan, 1995). We found that within the electron temperature range 300 $6000 \mathrm{~K}$. the new energy transfer rates and the electron cooling rate obtained by using the Allan (1995) vibrational cross sections are much larger than those of Prasad and Furman (1973) obtained by using the Linder and Schmidt (1971) data. We have shown that the relative contribution of the vibrational levels $v=5,6,7$ to the electron cooling rate and the production frequency of the $\mathrm{O}_{2}$ vibrational quanta is less than $0.5 \%$ for $T_{e} \leq 6000 \mathrm{~K}$ and the vibrational levels $v=1-4$ are enough to calculate $L$ and $W$.

This ${ }^{2} \Pi_{g}$ resonance dominates the vibrational cross sections in $\mathrm{O}_{2}$ at energies below about $2.5 \mathrm{eV}$ while the ${ }^{4} \sum_{u}^{-}$and ${ }^{2} \sum_{u}^{-}$resonances of $\mathrm{O}_{2}$ give the major contribution to the $\mathrm{O}_{2}$ vibrational cross sections above 3-4 eV. We found that the effects of the ${ }^{4} \sum_{u}^{-}$and ${ }^{2} \sum_{u}^{-}$ resonances are negligible on the calculation of $Q_{0 v}, L$, and $W$ in comparison with those for the ${ }^{2} \Pi_{g}$ resonance. The results of this study also provide sufficient evidence to neglect the effects of the excitation of the $a^{1} \Delta_{g}$ and $b^{1} \sum_{g}^{+}$electronic states of $\mathrm{O}_{2}$ with thermal electrons on the calculation of $T_{e}$.

In this study, our goal was also to evaluate the accuracy of the electron cooling rate due to rotational excitation of $\mathrm{O}_{2}$ found by Dalgarno et al. (1968). As we have shown, this currently accepted rate of electron energy loss associated with rotational transitions in $\mathrm{O}_{2}$ must be decreased by a factor of 13 .

Acknowledgements. The research described in this publication was supported by grant 96-05-64031 from the Russian Foundation of the Fundamental Researches. I would like to thank the anonymous referees for detailed and constructive comments.
Topical Editor D. Alcaydé thanks T.G. Slanger and G.J. Kockarts for their help in evaluating this paper.

\section{References}

Albritton, D. L., I. Dotan, W. Lindinger, M. McFarland, J. Tellinghuisen, and F. C. Fehsenfeld, Effects of ion speed distributions in flow-drift tube studies on ion-neutral reactions, J. Chem. Phys., 66, 410-421, 1977.

Allan, M.,Measurement of absolute differential cross sections for vibrational excitation of $\mathrm{O}_{2}$ by electron impact, J. Phys. B., 28, 5163-5175, 1995

Dalgarno, A., McElroy, M. B., and J. C. G. Walker, The effect of oxygen cooling on ionospheric electron temperatures, Planet. Space Sci., 16, 1371-1380, 1968.

Gerjuoy, E. and S. Stein, Rotational excitation by slow electrons, Phys. Rev. A, 97, 1671-1679, 1955.

Hake, R. D., Jr., and A. V. Phelps, Momentum- transfer and inelastic-collision cross sections for electrons in $\mathrm{O}_{2}, \mathrm{CO}$, and $\mathrm{CO}_{2}$, Phys. Rev., 158, 70-84, 1967.

Hedin, A.E., MSIS-86 thermospheric model, J. Geophys. Res., 92, 4649-4662, 1987.

Hierl, M. P., I. Dotan, J. V. Seeley, J. M. Van Doren, R. A. Morris, and A. A. Viggiano, Rate constants for the reactions of $\mathrm{O}^{+}$with $\mathrm{N}_{2}$ and $\mathrm{O}_{2}$ as a function of temperature $(300-1800 \mathrm{~K})$, J. Chem. Phys. 106, 3540-3544, 1997.

Itikawa, Y., M. Hayashi, H. Nishimura, and S. Tsurubuchi, Cross sections for collisions of electrons and photons with oxygen molecules, J. Phys. Chem. Ref. Data, 18, 23-42, 1989.

Lane, N. F. and A. Dalgarno, Electron cooling by vibrationally excitation of $\mathrm{O}_{2}, J$. Geophys. Res., 74, 3011-3012, 1969.

Lawton, S. A. and A. V. Phelps, Excitation of the $b^{1} \sum_{\mathrm{g}}^{+}$state of $\mathrm{O}_{2}$ by low energy electrons, J. Chem. Phys., 69, 1055-1068, 1978.

Linder, F. and H. Schmidt, Experimental study of low energy e- $\mathrm{O}_{2}$ collision processes, Z- Naturforsch. 26 A, 1617-1625, 1971.

Lopez-Gonzalez, M. J., Lopez-Moreno, J. J., Lopez-Valverde, M. A., and R. Rodrigo, Behaviour of the $\mathrm{O}_{2}$ infrared atmospheric $(0-0)$ band in the midle atmosphere during evening twilight and in night, Planet. Space Sci., 37, 61-72, 1989.

McFarland, M., D. L. Albritton, F. C. Fehsenfeld, E. E. Ferguson, and A. L. Schmeltekopf, Flow-drift technique for ion mobility and ion-molecule reaction rate constant measurements, II. Positive ion reaction of $\mathrm{N}^{+}, \mathrm{O}^{+}$, and $\mathrm{N}_{2}^{+}$with $\mathrm{O}_{2}$ and $\mathrm{O}^{+}$with $\mathrm{N}_{2}$ from thermal to $2 \mathrm{eV}$, J. Chem. Phys., 59, 6620-6628, 1973.

Mentzoni, M. H., and R. V. Row, Rotational excitation and electron relaxation in nitrogen, Phys. Rev., 130, 2312-2316, 1963.

Middleton, A. G., Teubner, P. J. O., and M. J. Brunger, Experimental confirmation for resonance enhancement in the $a^{1} \Delta_{g}$ and $b^{1} \sum_{g}^{+}$states of $\mathrm{O}_{2}$, Phys. Rev. Lett., 69, 2495-2498, 1992.

Noble, C. J., Higgins, K., Woste, G., Duddy, P., Burke, P. G., Teubner, P. J. O., Middleton, A. G., and M. J. Brunger, Resonant mechanisms in the vibrational excitation of ground state $\mathrm{O}_{2}$, Phys. Rev. Lett., 76, 3534-3537, 1996.

Pavlov, A. V., On the $\mathrm{O}_{2}^{*}$ content in the upper atmosphere and the effect of $\mathrm{N}_{2}^{*}$ and $\mathrm{O}_{2}^{*}$ on the electron density (in Russian), Geomagn. Aeron., 29, 818-823, 1989.

Pavlov, A. V., The role of vibrationally excited oxygen and nitrogen in the D and E regions of ionosphere, Ann. Geophysical, 12, 1085-1090, 1994.

Pavlov, A. V., The role of vibrationally excited oxygen and nitrogen in the ionosphere during the undisturbed and geomagnetic storm period of 6-12 April 1990, Ann. Geophysical, 16, in press, 1998.

Pavlov, A. V., Buonsanto, M. J., Schlesier, A. C. and P. G. Richards, Comparison of models and data at Millstone Hill during the June 5-11, 1991, storm, J. Atmos. Terr. Phys., 60, in press, 1998

Prasad, S. S. and D. R. Furman, Electron cooling by molecular oxygen, J. Geophys. Res., 78, 6701-6707, 1973. 
Radzig, A. A., and B. V. Smirnov, The reference book in atomic and molecular physics (in Russian), Atomizdat, Moscow, 1980.

Shyn, T. W., and C. J. Sweeney, Vibrational-excitation cross sections of molecular oxygen by electron impact, Phys. Rev. A, 48, 1214-1217, 1993.

Smith, D., and A. G. Dean, Electron temperature relaxation rates in flowing afterglow plasmas containing molecular nitrogen and oxygen, J. Phys. B., 8, 997-1008, 1975.

Stubbe, P., and W. S. Varnum, Electron energy transfer rates in the ionosphere, Planet. Space Sci., 20, 1121-1126, 1972.
Trajmar, S., Cartwright, D. C., and W. Williams, Differential and integral cross sections for the electron-impact excitation of the $a^{1} \Delta_{g}$ and $b^{1} \sum_{g}^{+}$states of $\mathrm{O}_{2}$, Phys. Rev. A., 4, 1482-1492, 1971.

Wong, S. F., Boness, M. J. W., and G. J. Schulz, Vibrational excitation of $\mathrm{O}_{2}$ by electron impact above $4 \mathrm{eV}$, Phys. Rev. Lett., 31, 969-972, 1973.

Yee, J. -H., Crowley, G., Roble, R. G., Skinner, W. R. Burrage, M. D. and P. B. Hays, Global simulation and observations of $\mathrm{O}\left({ }^{1} \mathrm{~S}\right), \mathrm{O}_{2}\left({ }^{1} \sum\right)$ and $\mathrm{OH}$ mesospheric nightglow emissions, $J$. Geophys. Res., 102, 19949-19968, 1997. 